\title{
Orbital variation of the rotating non-spherical dust grains due to change of the solar radiation forces by shape effect
}

\author{
Md. Abu Saklayen and Tadashi Mukai \\ Garduate School of Science and Technology, Kobe University, Nada 657-8501, Kobe, Japan
}

(Received December 26, 2003; Revised March 29, 2004; Accepted May 31, 2004)

\begin{abstract}
We present the values of a ratio $\beta$ of the solar radiation pressure force to the solar gravity on the finite circular cylindrical grains, as functions of an aspect ratio of the cylinder and an incident angle $\Theta$ of the solar radiation. By using the resulting formula of $\beta(\Theta)$, the trajectory of the Kepler orbit for the rotating silicate cylinder is computed associated with a spin motion under the assumption that the spin axis is along the shortest axis of the cylinder and points to the direction perpendicular to the solar radiation. We found for the silicate cylinder grain with a mass equivalent to a sphere with a radius of $0.15 \mu \mathrm{m}$, and the aspect ratio of 2.0 that a heliocentric distance of the grain varies periodically with a time, having an amplitude of the fluctuation in the heliocentric distance of about $0.02 \mathrm{AU}$, where the spin velocity is 0.25 rotation/day and the initial orbit has a semi-major axis $3.0 \mathrm{AU}$ and an eccentricity 0 . In addition, during such a fluctuation of the heliocentric distance, the instantaneous eccentricity of the orbit also varies simultaneously from 0 to 1.6 with the rotation of the grain. This implies that the in-situ measurements of orbital elements of impact grains on the dust detector may record those instantaneous orbital elements related to the phase of the grain's rotation.
\end{abstract}

Key words: Interplanetary dust, irregularly shape, solar radiation forces, spin motion, dynamics, orbital evolution.

\section{Introduction}

It is widely accepted that the asteroids supply the interplanetary dust grains. A discovery of the asteroid dust bands by the Infrared Astronomical Satellite (IRAS) associated with the Koronis and Themis families (Low et al., 1984) has proved that the collisional debris from the asteroids becomes scattered through the interplanetary space. Further satellite observations by the Diffuse Infrared Background Experiment (DIRBE) instrument on the Cosmic Background Explorer (COBE) (Spiesman et al., 1995), as well as the ground-based observations in the visible (Ishiguro et al., 1998), have confirmed the presence of the dust bands. Reach, Franz and Weiland (1997) have suggested that the dust grains resulting from the gradual erosion by collisions among the family's members really form the dust band structure in the interplanetary space.

On the other hand, the laboratory experiments for collisions of solid bodies, e.g. Fujiwara and Tsukamoto (1981), suggest that the collisional debris has an irregularly shape, and is in the rotational state. These results predict that the collisional debris from the asteroids has a non-spherical shape and is in the state of rotation. To study the dynamical evolution of collisional debris after releasing from the parent asteroid, we should examine the effect of solar radiation forces on the ejected dust grain, taking into account its shape and rotation state.

The strength of solar radiation pressure force on the dust grain is proportional to the effective cross section of the

Copy right (c) The Society of Geomagnetism and Earth, Planetary and Space Sciences (SGEPSS); The Seismological Society of Japan; The Volcanological Society of Japan; The Geodetic Society of Japan; The Japanese Society for Planetary Sciences; TERRAPUB. grain, while the solar gravitational force is proportional to its mass. This suggests that the projected-area to mass ratio is a key parameter to understand the dynamical behavior of dust grain in the interplanetary space. If we consider the situation when the projected area to mass ratio changes with the spin motion of the grain, a time variation of $\beta$ arises, where $\beta$ is a ratio of the solar radiation force to the solar gravity on the grain. This variation of $\beta$ would lead to the periodic fluctuation of the Kepler orbit of the grain, associated with its rotational state. It is our motivation to prove this prediction quantitatively.

In this paper, we assume that the dust grain has a finite circular cylindrical shape as a simple example of non-spherical shapes to emphasize the importance of the shape effect on the solar radiation pressure. The values $\beta$ of the cylindrical grain are derived in Section 2 based on the DDA method (e.g. Draine and Flatau, 1994), as functions of the aspect ratio of the cylinder and the incident angle of the solar radiation to the axis of the symmetry. A periodic fluctuation of the $\mathrm{Ke}$ pler orbit of such a cylindrical grain due to the change of $\beta$ with its rotation is examined in Section 3. Discussion is done in Section 4 and conclusions are summarized in Section 5.

\section{The $\beta$ Value for Non-Spherical Grain 2.1 A definition of $\beta$}

The radiation pressure force on small grain strongly depends on the physical properties of the grain. Burns et al. (1979) have reviewed the interactions of the solar radiation with small spherical grain, and shown the value of $\beta$ computed for the grain with different kind of grain materials, as a function of its radius. It is generally shown that the $\beta$ value for absorbing materials exceeds unity in some region of grain 
(a)

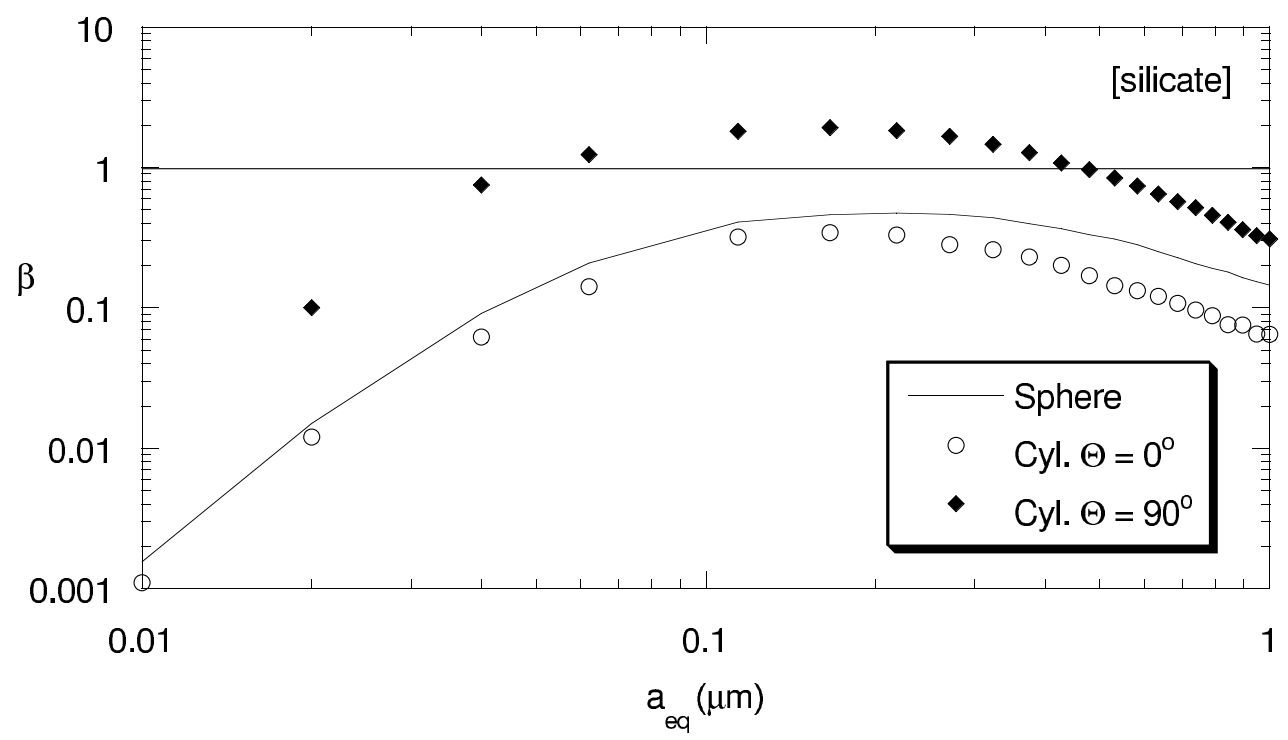

Fig. 1(a). A ratio of $\beta$ of the solar radiation pressure to the solar gravity on a silicate grain. The $\beta$ values are derived for the spherical grain with a radius $a_{e q}$ (the solid curve) and the circular cylinder with a mass equivalent to the sphere with a radius $a_{e q}$ and with an aspect ratio 2.0. Two incident angles of the solar radiation are shown, i.e. parallel $\left(\Theta=0^{\circ}\right.$, open circles $)$ and perpendicular $\left(\Theta=90^{\circ}\right.$, filled diamonds $)$ to the symmetry axis of the cylinder.

radius, while that for dielectric materials never exceeds unity.

The radiation pressure force due to solar radiation on a grain with a geometrical cross section $A$ is expressed as

$$
F_{\text {rad }}=\left(\frac{A}{c}\right)\left(\frac{R_{0}}{r}\right)^{2} \int_{0}^{\infty} B_{\odot}(\lambda) Q_{p r}\left(s, m^{*}, \lambda\right) d \lambda
$$

where $r$ is a heliocentric distance of the grain, and $R_{0}$ and $B_{\odot}$ denote the solar radius and its spectrum, respectively. The $c$ is a speed of light, $\lambda$ is a wavelength of radiation and $Q_{p r}$ is an efficiency factor of radiation pressure. The value of $Q_{p r}$ depends on the grain size $s$ and the optical constant $m^{*}$ of grain material, as well as the grain shape.

Our ability to compute the radiation pressure force on the solid grain with arbitrary shape was limited because of a lack of rigorous solution for the interaction between the light and the irregularly shaped grain. Therefore, it was assumed in most of the previous works (e.g. Burns et al., 1979) that the dust grain is a sphere for simplicity, and Mie theory is applied to solve the light scattering problems. For nonspherical grains, the rigorous analytic solutions exist only for some special cases, e.g. that for a homogeneous spheroid in arbitrary orientation presented by Asano and Yamamoto (1975; errata in Asano and Yamamoto, 1976). Purcell and Pennypacker (1973) developed the "discrete dipole approximation" (DDA) method, where the scattered particle is represented by an array of $N$ points dipoles with spacing between the dipoles smaller than the wavelength of interest. The DDA is a very flexible and general technique for calculating the optical properties of arbitrary shaped grains, as mentioned in Draine and Flatau (1994). We will use the DDA to compute the value of the radiation pressure force on the finite circular cylinder grain in this paper.

The gravitational attraction force on the grain with mass $m$ is defined by

$$
F_{g}=\frac{G M_{\odot} m}{r^{2}}
$$

where $G$ is a gravitational constant and $M_{\odot}$ is the mass of the Sun. Consequently, a value of $\beta$ is derived from

$$
\beta=F_{\text {rad }} / F_{g}=C\left(\frac{A}{m}\right) \int_{0}^{\infty} B_{\odot}(\lambda) Q_{p r}\left(s, m^{*}, \lambda\right) d \lambda
$$

where $C$ is a constant of $1.09 \times 10^{6}$ in cgs unit. The values of the solar spectrum $B_{\odot}(\lambda)$ are adapted from the data compiled in Mukai (1989).

\subsection{Computed results of $\beta$ for a finite circular cylindri- cal grain}

We will take for calculations of $\beta$ a finite circular cylinder with an aspect ratio $a / b$, where $a$ is a half length of the curving side of the cylinder and $b$ is a radius of its circular end. For the material of cylindrical grain, silicate and magnetite are assumed. The values of mass density are $2.4 \mathrm{~g} \mathrm{~cm}^{-3}$ for silicate and $5.2 \mathrm{~g} \mathrm{~cm}^{-3}$ for magnetite, and the optical constants $m^{*}$ for silicate and magnetite are derived from the data compiled in Mukai (1989). It should be noted that we do not request the presence of silicate and magnetite in the interplanetary dust grains. They only represent the dielectric and absorbing materials in space.

It is expected that in the derivation of $\beta$ for the cylindrical grain, the incident angle of radiation $\Theta$ plays an important role, where $\Theta$ is defined as an angle between the solar radiation and the long symmetry axis of the cylinder. That is, $\Theta=0^{\circ}$ denotes that the solar radiation comes from the perpendicular direction to the circular end of the cylinder, while $\Theta=90^{\circ}$ means that the radiation comes perpendicularly to the curving side of the cylinder.

Figures 1(a) and 1(b) show a comparison of $\beta$ of silicate and magnetite grain, respectively, as a function of an equivalent radius $a_{e q}$ for the sphere and the cylinder with an aspect ratio $a / b=2.0$, where $a_{e q}$ is defined as a radius of the sphere with the equivalent mass to the cylinder of interest. To compute the $\beta$ value for the cylindrical grain with $a_{e q}$ from 0.01 


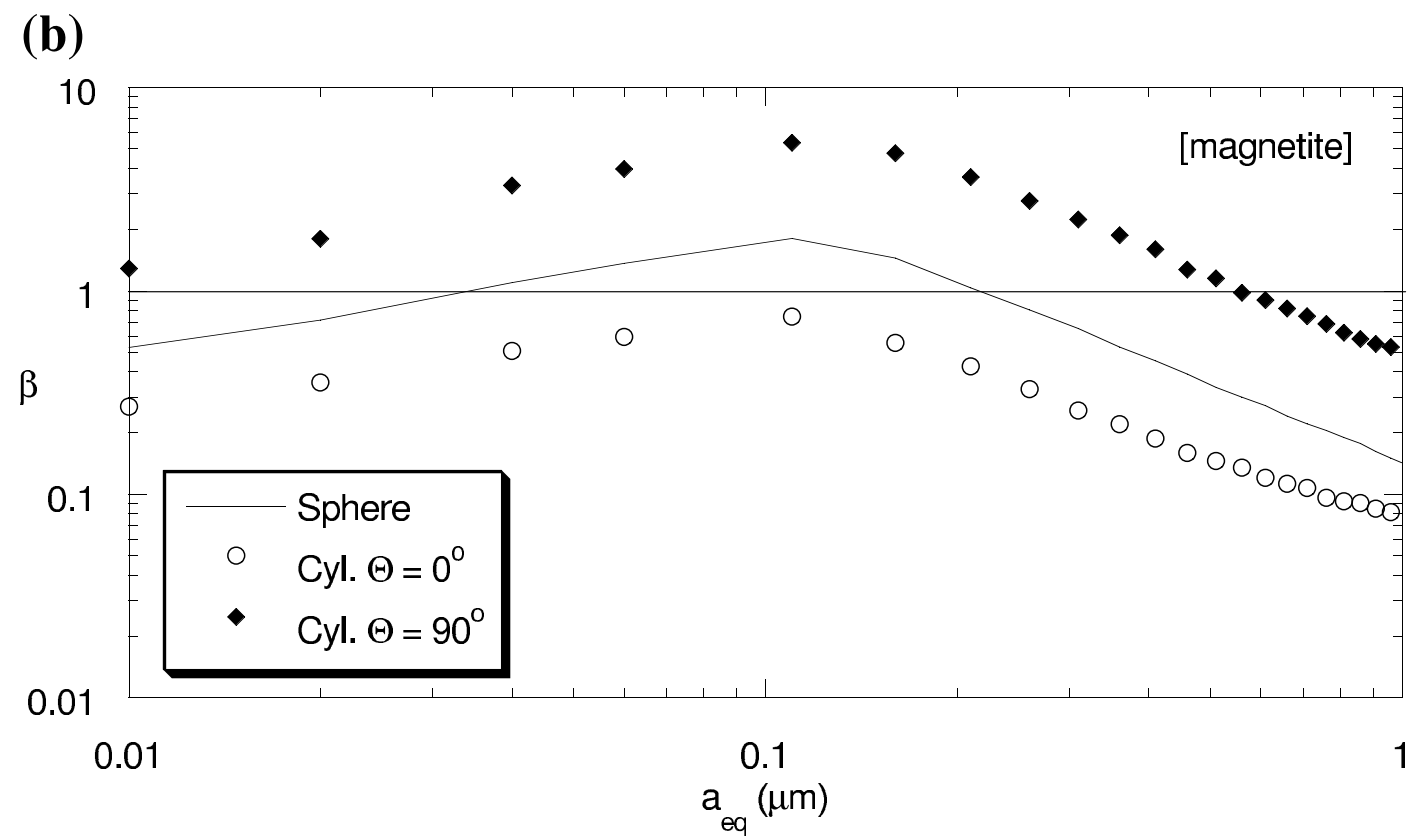

Fig. 1(b). Same as Fig. 1(a), but for a magnetite grain.

$\mu \mathrm{m}$ to $1 \mu \mathrm{m}$, we apply the DDA method (Draine and Flatau, 2000). We change the number of dipoles $N$ to satisfy the applicability criterion of the DDA, i.e. $\left|m^{*}\right| k_{0} d<1$, where $k_{0}=2 \pi / \lambda$ and $d$ is the spacing between two dipoles. We take $N=12640$ for $a_{e q}=0.15 \mu \mathrm{m}$. This value of $N$ was set under the condition that a variation of $Q_{p r}$ computed by the DDA with increasing $N$ becomes negligibily small. The $\beta$ values for the sphere were computed by the DDA, and have been checked that they agree very well with those computed by Mie theory.

For the cylindrical grain, we found that the $\beta$ value strongly depends on the incident angle of radiation $\Theta$ (see Figs. 1). Since in $a / b=2.0$ the geometrical cross-section for $\Theta=90^{\circ}$ is larger than that for $\Theta=0^{\circ}$ and for the sphere with equivalent mass, then the $\beta$ value for $\Theta=90^{\circ}$ becomes always larger than other cases. In addition, in $\Theta=90^{\circ}$ it is found that the $\beta$ value for silicate grain exceeds unity in $0.052 \mu \mathrm{m}<a_{e q}<0.5 \mu \mathrm{m}$, whereas for the spherical grain and the cylinder in $\Theta=0^{\circ}$, the $\beta$ values never exceed unity. It is well known (see e.g. Burns et al., 1979 and Mukai, 1989) that for a spherical dielectric grain (silicate and water-ice) the $\beta$ value is always less than unity. It is shown, however, that the irregularly shaped dielectric grain has the $\beta$ value larger than unity in some cases mentioned above. On the other hand, it is also found that the $\beta$ value for magnetite grain in $\Theta=0^{\circ}$ never exceeds unity. It is believed that the spherical grain consisting of the absorbing materials, such as magnetite, has the $\beta$ larger than unity in the range of grain radius less than $1 \mu \mathrm{m}$. Therefore, the appearance of the absorbing grains with $\beta<1$ in all size range should be noted as a shape effect of the solar radiation pressure force on the grain.

We show the dependence of $\beta$ for the cylinder on its aspect ratio $a / b$, where the silicate and magnetite cylinders with $a_{e q}=0.15 \mu \mathrm{m}$ are taken as test samples. It is known that the spherical grains of silicate and magnetite with a radius
$0.15 \mu \mathrm{m}$ have, respectively, $\beta=0.544$ and 1.84 (see Mukai 1989). It is found in Fig. 2 that for silicate grain, the $\beta$ value in $\Theta=0^{\circ}$ decreases with increasing $a / b$ and goes below unity in $a / b \geq 0.42$, while $\beta$ in $\Theta=90^{\circ}$ increases with $a / b$ and exceeds unity in $a / b \geq 1$. On the other hand, for magnetite grain, $\beta \leq 1$ occurs in $a / b \leq 0.15$ in $\Theta=90^{\circ}$ and in $a / b \geq 1.5$ in $\Theta=0^{\circ}$. These evidence can be explained from the fact that the geometrical cross section $A$ of the cylinder changes with $a / b$ when the volume of the cylinder is constant. That is, in $\Theta=0^{\circ}, A=\pi b^{2} \sim(a / b)^{-2 / 3}$ whereas in $\Theta=90^{\circ}, A=4 a b \sim(a / b)^{1 / 3}$. We found that a slightly deviation of $\beta$ from this expectation occurs due to a shape effect in $Q_{p r}$ deduced from the DDA.

The $\Theta$ dependence of $\beta$ is also examined for the cylinder with $a_{e q}=0.15 \mu \mathrm{m}$ and $a / b=2.0$, for silicate and magnetite (see Fig. 3). From the curve fitting to the computed $\beta$ values, we have derived a relation of

$$
\beta(\Theta)=\beta_{0}+K \sin ^{2} \Theta
$$

where $\beta_{0}$ is $\beta$ at $\Theta=0^{\circ}$ and $K$ is a constant, i.e. $\beta_{0}=0.28$ and $K=1.55$ for silicate and $\beta_{0}=0.76$ and $K=4.6$ for magnetite.

\section{Dynamical Behavior of a Rotating Cylindrical Grain}

3.1 The equation of motion

Some efforts have been done to treat the dynamical behaviour of the non-spherical dust grain. Namely, Voshchinnikov and Il'in (1983) examined the radiation pressure forces on cylindrical dust grains, and Gustafson (1989) studied the dynamics of non-spherical dust in the interplanetary space. Il'in and Voshchinnikov (1998) calculated analytically the radiation pressure force, around a red giant star, acting on a spheroid (prolate and oblate). They found that for a small spheroid with the equivalent radius $a_{e q}$ less than 


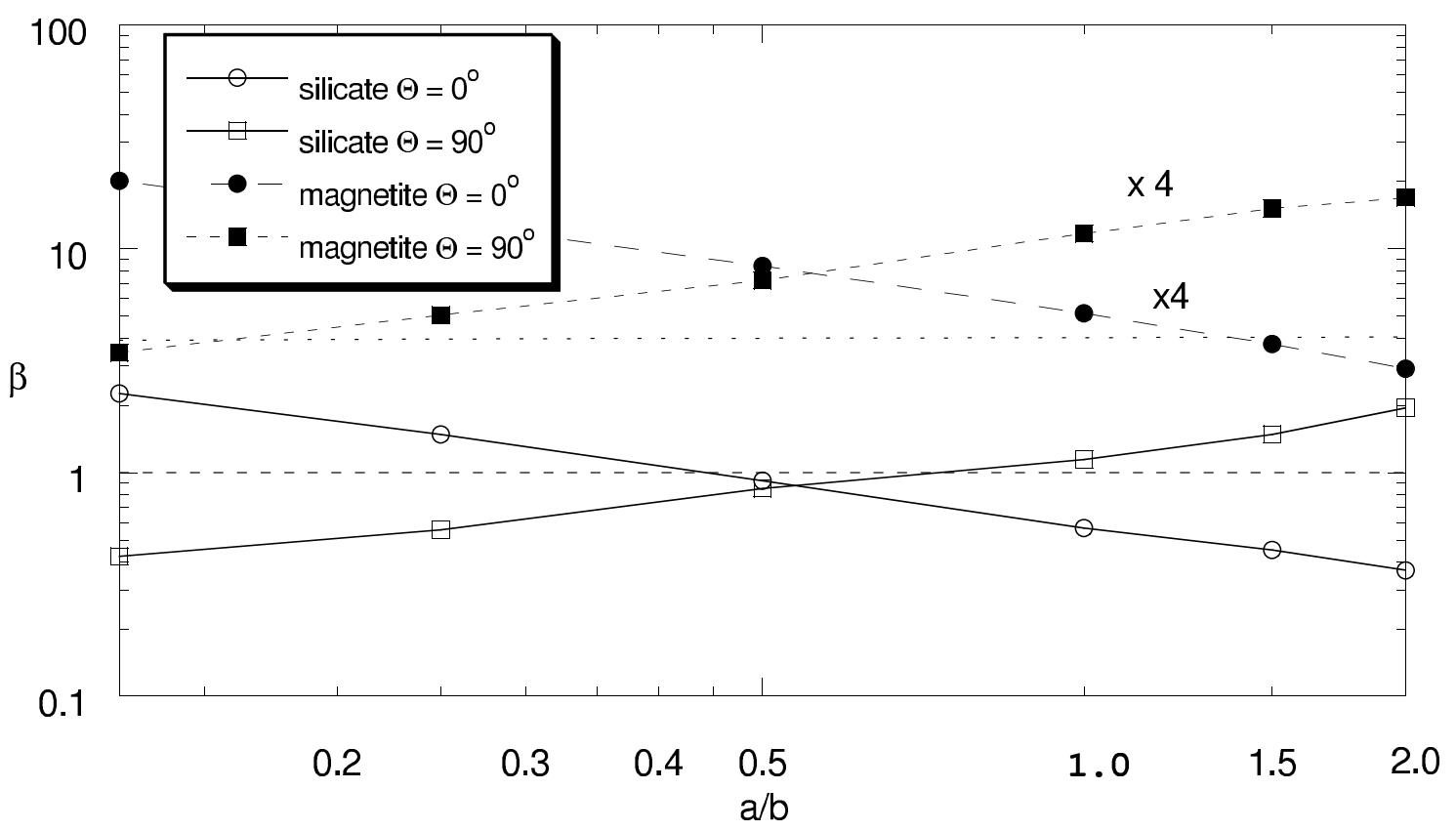

Fig. 2. $\beta$ vs. an aspect ratio $a / b$ of the circular cylinder grain with $a_{e q}=0.15 \mu m$, consisting of silicate (open marks) and magnetite (filled marks). Two incident angles $\Theta=0^{\circ}$ and $90^{\circ}$ are taken. Note the offset in $\beta$ of the magnetite.

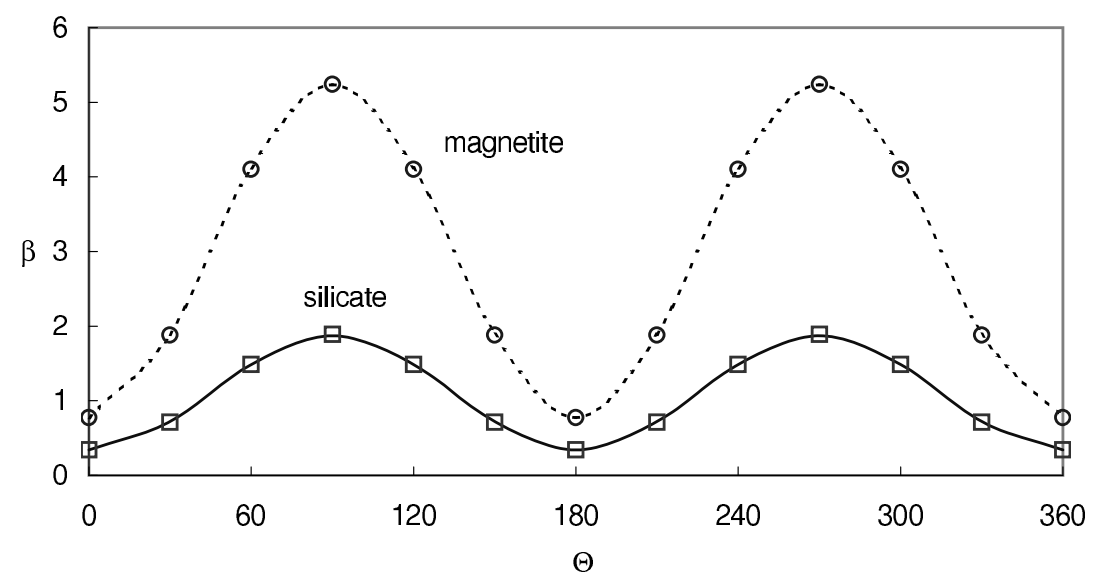

Fig. 3. $\beta$ vs. an incident angle $\Theta$ of the solar radiation for the circular cylinder grains with silicate (solid curve with open squares) and magnetite (dotted curve with open circles), where $a_{e q}$ is $0.15 \mu \mathrm{m}$ and the aspect ratio is 2.0 .

$0.1 \mu \mathrm{m}$, the radiation pressure force is greater than that for a sphere with equivalent mass. Mukai et al. (1992) have examined the interaction of solar radiation with irregularly shaped fluffy aggregates. They found that the $\beta$ decreases as the porosity of aggregate increases. Furthermore, it is shown that the values of $\beta$ for a fluffy aggregate computed based on Mie theory and Maxwell-Garnett effective medium theory well agree with those derived by the DDA. Kimura et al. (2002) have extended the analysis for the dynamical effect of solar radiation on the fluffy dust grains based on the DDA method.

Although many trials to study the dynamics of nonspherical grains in the interplanetary space have been done previously as mentioned above, no quantitative examinations of the coupling between the heliocentric Kepler motion and the rotation of the grain have appeared. We will firstly present the coupling effect of spin motion with the Kepler motion of the grains in this paper.
For a heliocentric grain with velocity $\mathbf{v}$ under the influence of solar gravity and solar radiation pressure, the equation of motion becomes,

$$
\dot{\mathbf{v}}+G M_{0}(1-\beta)\left[(1-\dot{r} / c) \mathbf{r}_{0}-\mathbf{v} / \mathrm{c}\right] / \mathrm{r}^{2}=0,
$$

where $\dot{\mathbf{v}}$ means an acceleration vector, and $\mathbf{r}_{0}$ denotes the unit heliocentric radius vector. The $\dot{r}$ means a radial component of $\mathbf{v}$. The velocity dependent portion of Eq. (5) is called the Poynting-Robertson drag.

It has been shown in Eq. (4) that the value of $\beta$ for the cylindrical grain varies with the incident angle of radiation $\Theta$. When we assume that the grain rotates with a spin velocity $\omega$ along the axis perpendicular to the curving side of the cylinder and its spin axis is normal to the heliocentric radial direction, the equation (4) is written as,

$$
\beta(\omega t)=\beta_{0}+K \sin ^{2}(\omega t)
$$

where $\Theta=0^{\circ}$ at a time $t=0$. 


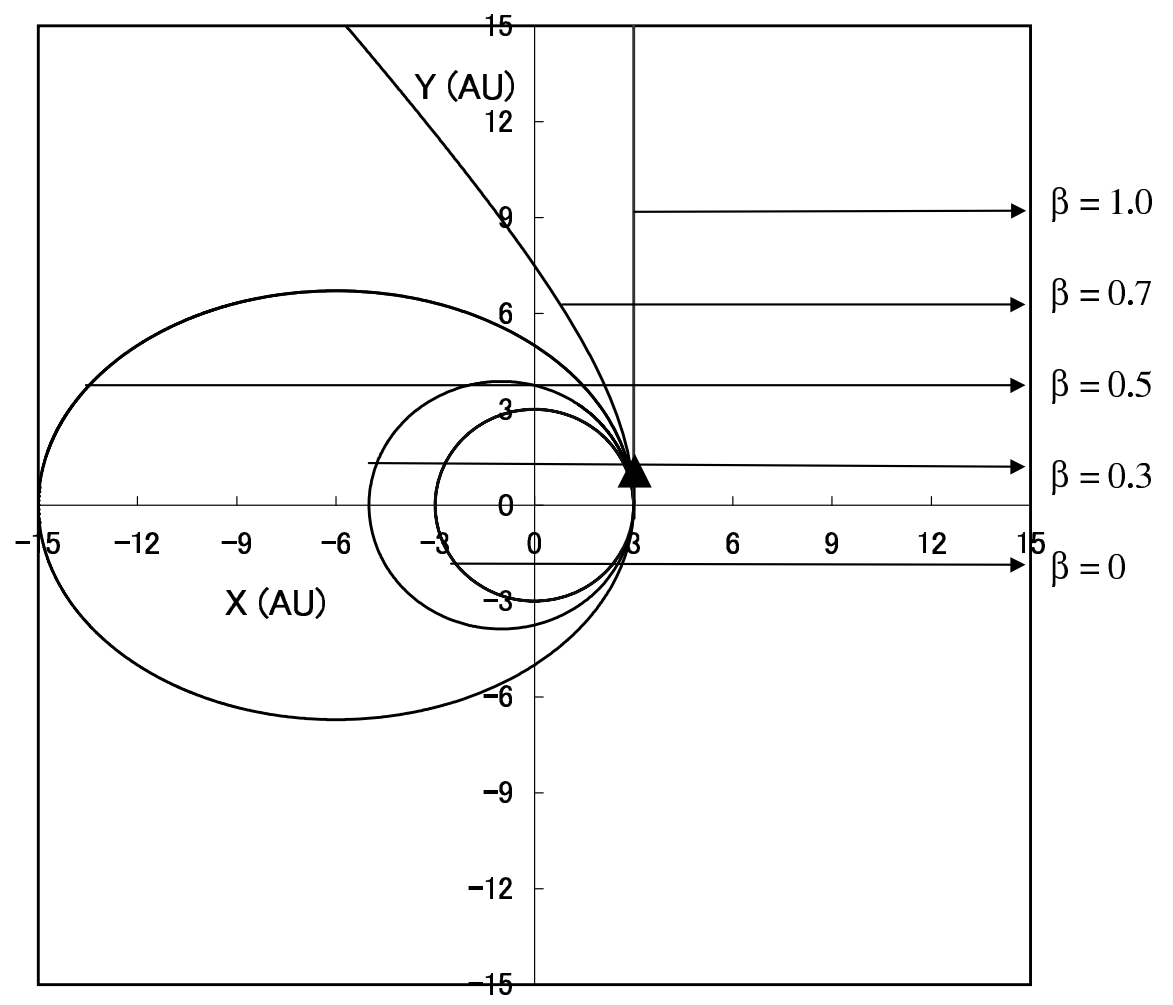

Fig. 4. Trajectory of a grain with a $\beta$ value, released from the parent body on the circular Kepler orbit with a semi-major axis of 3 AU, where $\beta$ is a ratio of the solar radiation pressure force to the solar gravity on the grain. The initial position is at $X=3 \mathrm{AU}$ and $Y=0$, and the direction of motion is toward the $+Y$ direction

The initial condition for the numerical simulation of the rotating cylindrical grain is assumed that the grain is released from the parent body, which is on the circular orbit of the semi-major axis of $3 \mathrm{AU}$ and the inclination of $0^{\circ}$. We neglect a relative velocity of the grain to the parent body. The RADAU numerical integrator (Everhart, 1985) is used to solve Eqs. (5) and (6) simultaneously.

\subsection{Fluctuation of the Kepler orbit}

After releasing from the parent body, the grain feels a relatively low gravitational attraction by a factor $1-\beta$. Consequently, its orbit differs from that of the parent body. Figure 4 shows the orbits of the grains with different $\beta$ values released from the circular Kepler prograde orbit at a heliocentric distance $r=3$ AU on the $X$ axis. As $\beta$ increases, the resulting orbit of the grain has higher eccentricity. The perihelion position of the orbit is the same as the starting position in the $X$ axis because the Poynting-Robertson effect which changes the perihelion distance of the orbit, plays no significant role during the time scale of interest (nearly one Kepler period at $3 \mathrm{AU})$.

To pursue the trajectory of the rotating cylinder, we solve Eqs. (5) and (6) simultaneously for a test grain of a silicate cylinder with $a_{e q}=0.15 \mu \mathrm{m}$ and $a / b=2.0$. Figure 5 shows the periodic variation of heliocentric distance $r$ with a time, associated with the spin motion, during 5 days after 25 days leaving from the parent body. Note that there is no physical meaning to select " 25 days after leaving" except to emphasize the difference of the minimum heliocentric distance of rotating cylinder from $r=3 \mathrm{AU}$. It is found that the grain with slow spin velocity shows wider change in heliocentric distance than the grain with fast spin velocity.
The closest distance of the rotating grain to the Sun increases with a time gradually by the effect of $\beta$ value. Furthermore, it is derived in Fig. 6 that the instantaneous eccentricity $e$ of the orbit for the rotating cylindrical grain varies periodically with its spin motion. Figure 6 shows that the grain with slower rotation velocity than $\omega=1$ rotation $/$ day changes its state of motion from a bound orbit to the unbound orbit and vise versa continuously, whereas the faster spin grains are always on the bound orbit.

The resulting orbits of the rotating cylindrical grains in the $X-Y$ plane are shown in Fig. 7(a). In Fig. 7(b), the enlargement of the variation in the part of the orbits is illustrated to show the fluctuation of the trajectory clearly for two cases, i.e., 0.25 rotation $/$ day and 0.5 rotation $/$ day. We found that the orbit of the cylindrical grain with $\omega=0.25$ rotation/day makes a band structure with a width of 0.02 $\mathrm{AU}$, while that with faster $\omega=2$ rotations $/$ day shows a narrow band structure with a width of 0.008 AU. The following relation between a width of the band structure $(\Delta r)$ and a spin velocity $(\omega)$ are derived, i.e.

$$
\Delta r(\mathrm{AU})=0.012-0.013 \log \omega(\text { rotation } / \text { day }) .
$$

In a case of a circular orbit of a parent body, the resulting band width does not depend on the released position of the rotating grain from the parent body. However, it is expected that the amplitude of fluctuation, i.e. the band width, is influenced by the initial position/velocity of the grain released from the parent body on an eccentric orbit. 


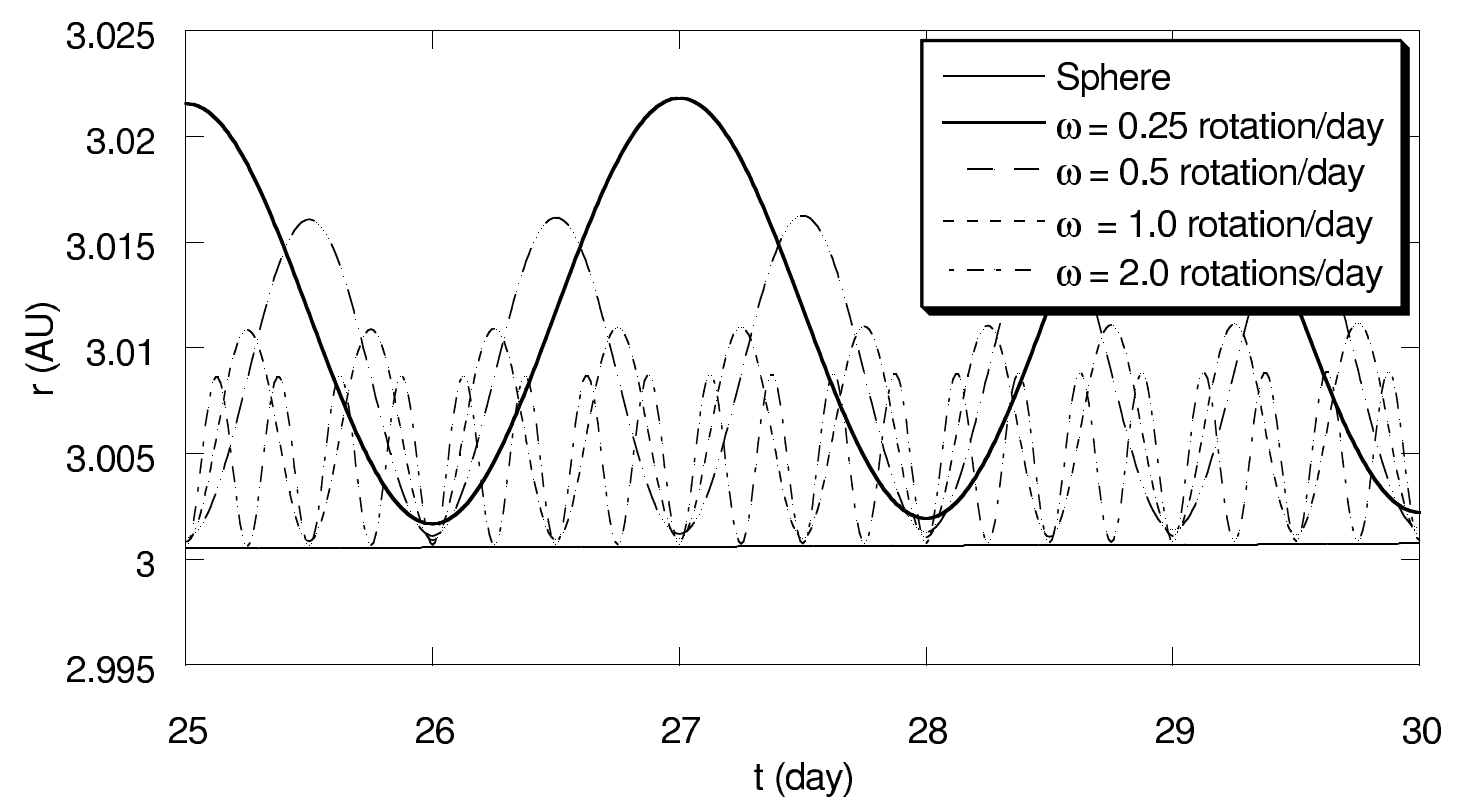

Fig. 5. Periodic variation of the heliocentric distance $r$ of the silicate grains during 5 days after 25 days from the released date, where four spin velocities $\omega$ are taken for the circular cylinder grains with $a_{e q}=0.15 \mu \mathrm{m}$ and the aspect ratio 2.0. For reference a sphere with $\beta=0.28$ is also shown by a thin solid curve.

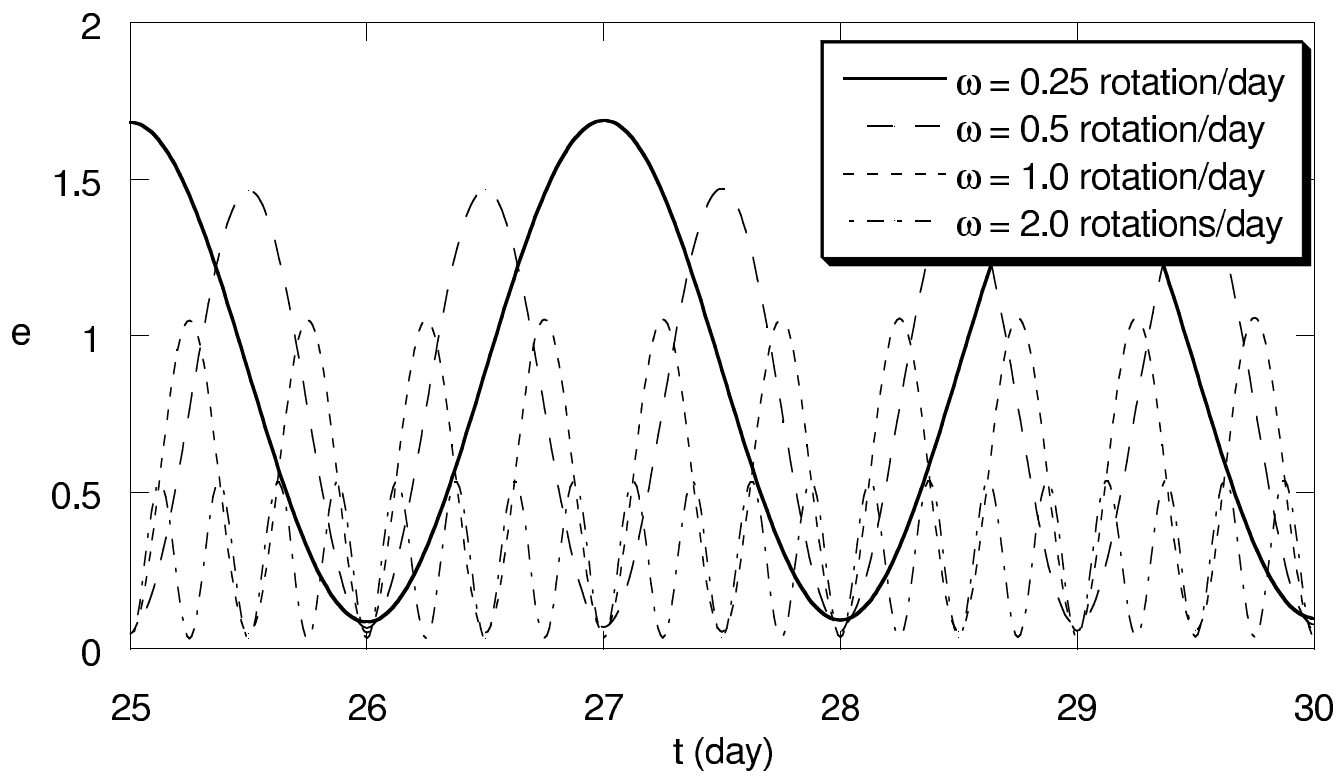

Fig. 6. Variation of the eccentricity $e$ with a time, which is obtained by the same simulation for the grain's motion done in Fig. 5 .

\section{Discussion}

It should be discussed about the presence of slow (1 rotation/day) spin grain. Although we have done no direct measurements of the spin velocity of interplanetary dust grains, it seems natural to expect that the interplanetary dust grains have spin motions obtained when they were produced by the catastrophic disruption by asteroids. Fujiwara and Tsukamoto (1981) have shown based on their laboratory measurements of catastrophic impacts into basaltic targets at a velocity of $2.7 \mathrm{~km} / \mathrm{s}$ that the spall fragments with sizes less than $1 \mathrm{~cm}$ have spin rate of larger than 1 rotation $/ \mathrm{s}$. It is shown that the spin rate increases with decreasing the size of fragment, though the lower limit of the detectable size is 0.4 $\mathrm{cm}$. From the limitation of the camera, the very low rotation rates could not be determined accurately. Consequently, no extremely slow spin grains (such as 1 rotation/day) could not be detected, even if they would exist in the ejected fragments.

Dohnanyi (1978) has reviewed the rotations of interplanetary dust grains, where he argued that the angular velocity of the fragments arisen in the catastrophic collisions will be distributed from zero to some maximum value related to the stress in fragmentation. Paddack and Rhee (1976) have examined the stabilization of spin motion for irregularly shaped metallic grain caused by the balance between the spin-up mechanism due to windmill effect by radiation pressure and the spin-down mechanism due to the magnetic spin damping effects by interplanetary magnetic field. They have shown 


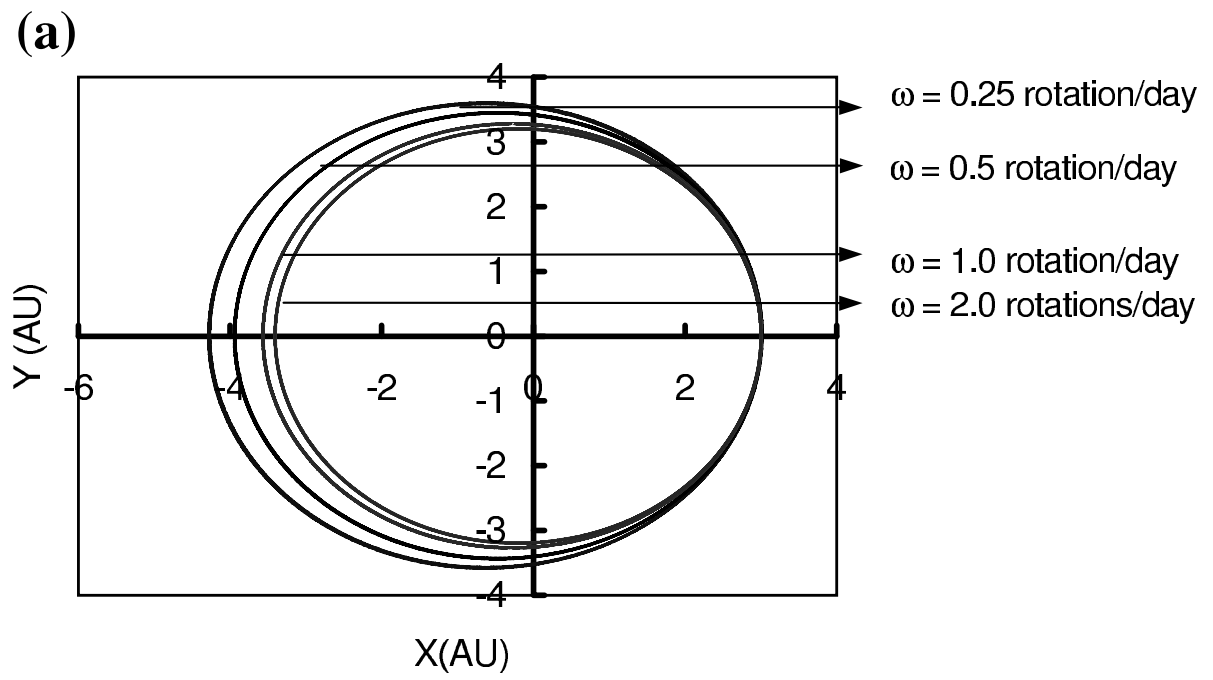

Fig. 7(a). The orbits in the $X-Y$ plane of cylindrical silicate grains with four spin velocities $\omega$, where the initial position is at $X=3$ AU and $Y=0$ on the circular prograde orbit with a semi-major axis of $3 \mathrm{AU}$.

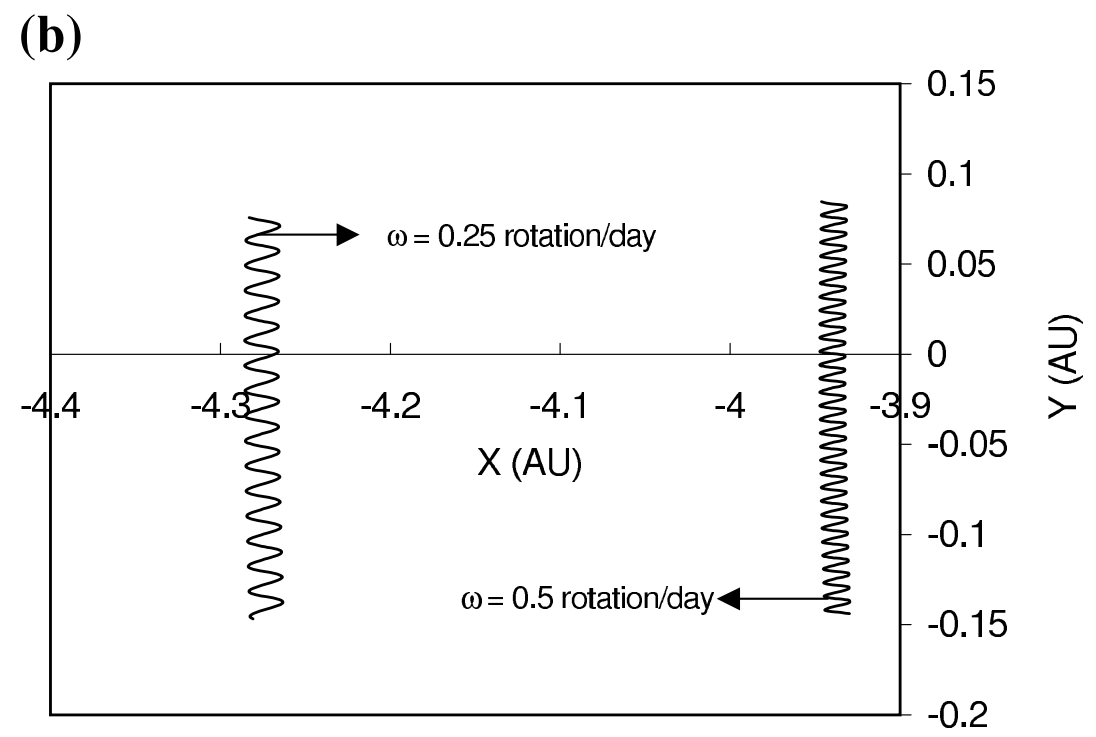

Fig. 7(b). Same as Fig. 7(a), but in a small scale in the $X-Y$ plane to show the fluctuation of the trajectory clearly.

that the lifetime of $\mu \mathrm{m}$ sized metallic grain against the rotational bursting is a few hundreds of years. On the other hand, Mukai (1981) has studied the spin-down effect of a spherical grain due to collisions by solar wind particles, and found that the rotating silicate grain with a radius less than $0.1 \mu \mathrm{m}$ would lose its spin angular momentum completely before it falls into the Sun by the Poynting-Robertson effect. This result suggests that the initially high spin grain gradually loses its spin velocity in the interplanetary space, and consequently the existence of very slow spin motion seems to be undeniable.

Concerning the alignment of spin vector with the normal direction to the solar radiation, several dynamical theories of grain orientation have been studied in the past (e.g. Greenberg, 1968). Gold mechanism (Gold, 1952) suggests that angular momentum imparted to a grain by collisions with the gas tends to be normal to the relative velocity. As a result, the elongated axis of a grain tends to be normal to its angu- lar momentum. This gas-dust interaction may happen at the event of catastrophic disruption of asteroids, and may lead to support our assumption that the spin vector is to normal to the symmetry axis of the cylinder. However, the available time scale for alignment in the disruption event seems to be too short to attain enough alignment of released grains. Since Davis-Greenstein mechanism (Davis and Greenstein, 1951) caused by an alignment torque due to paramagnetic relaxation requires the magnetic grains, then it may play a minor role in the silicate grain of interest.

It is natural to assume that the released grains from the parent body have arbitrary spin directions. Furthermore the directions of angular momentum, $\omega$ vector and one of the grain's principal axes of inertia are not all aligned. However, except the case that the spin axis of the grain is parallel to the solar radiation direction, a variation of the $\beta$ value with a spin motion occurs in some degree. As a result, the dynamical fluctuation of the Kepler orbit for irregularly shaped grain 
associated with its rotation motion is expected as a general feature for the grain efficiently affected by the solar radiation pressure force in the interplanetary space. It should be noted that the non-radial component of the radiation pressure force for non-spherical grains also plays some role in the dynamical evolution of such irregularly shaped grains for a fairly long time (e.g. Voshchinnikov and Il'in, 1983).

\section{Conclusions}

We have computed a ratio $\beta$ of the solar radiation pressure force to the solar gravity for the finite circular cylindrical grain with changing its aspect ratio $a / b$ and a direction of incident solar radiation $\Theta$. By using the resulting formula for a dependence of $\beta$ on $\Theta$, the trajectories of the rotating cylindrical grains in the solar system are computed for silicate grains. It is found that the heliocentric distance of the orbit for the slow rotating silicate grain $(\omega=0.25$ rotation/day) with a radius of the sphere with equivalent mass $a_{e q}=0.15 \mu \mathrm{m}$ and the aspect ratio $a / b=2.0$ varies periodically with a spin motion of the grain. Consequently the resulting orbit makes a band structure with a width of 0.02 AU. Although this width of the band structure is narrower one order of magnitude compared with the detected dust bands (see Ishiguro et al., 1998), the fluctuation effect of the orbit should be taken into account in the orbital evolution of the grains in the dust bands. In addition, during such fluctuation of the heliocentric distance of Kepler orbit, the instantaneous eccentricity of the orbit varies simultaneously from 0 to 1.6 with a time, associated with the spin motion of the grain. This implies that the in-situ measurement of the orbital elements of impinging grain into the dust counter may record such instantaneous orbital elements instead of the averaged orbital elements.

Acknowledgments. We thank B. Draine and P. Flatau for providing the original DDA code. We also thank Dr. N. Voshchinnikov and Dr. H. Ishimoto for their useful comments and suggestions. This research was supported by "The 21st Century COE Program of Origin and Evolution of Planetary Systems" in the Ministry of Education, Culture, Sports, Science, and Technology.

\section{References}

Asano, S. and G. Yamamoto, Light scattering by a spheroidal particle, Appl. Optics, 14, 29-49, 1975.

Asano, S. and G. Yamamoto, Light scattering by a spheroidal particle: ettratum, Appl. Optics, 15, 2028, 1976.

Burns, J. A., P. L. Lamy, and S. Soter, Radiation forces on small particles in the solar system, Icarus, 40, 1-48, 1979.

Davis, L. Jr. and J. L. Greenstein, The polarization of starlight by aligned dust grains Astrophys. J., 114, 206-215, 1951.
Dohnanyi, J. S., Particle Dynamics, in Comet Dust, edited by J. A. M. MacDonnell, pp. 527-587, Wiley, New York, 1978.

Draine, B. T. and P. J. Flatau, Discrete dipole approximation for scattering calculation, J. Opt. Soc. Am. A, 11, 1491-1499, 1994.

Draine, B. T. and P. J. Flatau, User Guide for the Discrete Dipole Approximation Code DDSCAT, http://xxx.lanl.gov./abs/astro-ph/0008151v3, 141, 2000.

Everhart, E., An Efficient Integrator that Uses Gauss=Radau Spacing, Dynamics of Comets: Their Origin and Evolution, Proc. of IAU Colloqu. 183, edited by A. Carusi and Gi. B. Valsecchi, pp. 185-192, Dordrecht: Reidel, Astrophysics and Space Science Library, 115, 1985.

Fujiwara, A. and A. Tsukamoto, Experimental study on the velocity of fragments in collisional breakup, Icarus, 44, 142-153, 1981.

Gold, T., Polarization of star light, Nature, 169, 322-324, 1952.

Greenberg, J. M., Interstellar Grains, in Nebulae and Interstellar Matter, edited by B. M. Middlehurst and L. H. Aller, pp. 221-364, The University of Chicago Press, Chicago \& London, 1968.

Gustafson, B. A. S., Comet ejection and dynamics of nonspherical dust particles and meteoroids, Astrophys. J., 337, 945-949, 1989.

Il'in, V. B. and N. V. Voshchinnikov, Radiation pressure on nonspherical dust grains in envelopes of late-type giants, Astron. Astrophys. Suppl. Ser., 128, 187-196, 1998.

Ishiguro, M., R. Nakamura, Y. Fujii, K. Morishige, H. Yano, H. Yasuda, S. Yokogawa, and T. Mukai, First detection of visible zodiacal dust bands from ground based observations, Astrophys. J., 511, 432-435, 1998.

Kimura, H., H. Okamoto, and T. Mukai, Radiation pressure and PoyntingRobertson effect for fluffy dust particles, Icarus, 157, 349-361, 2002.

Low, F. J., D. A. Beintema, T. N. Gautier, F. C. Gillett, C. A. Beichman, G. Neugebauer, E. Young, H. H. Aumann, N. Boggess, J. P. Emerson, H. J. Habing, M. G. Hauser, J. R. Houck, M. Rowan-Robinson, B. T. Soifer, R. G. Walker, and P. R. Wesselius, Infrared cirrus: New component of the infrared mission, Astrophys. J., 278, L19-L22, 1984.

Mukai, T., Spin-down Effect on an Interplanetary Dust Grain, Proc. of ISAS Lunar \& Planetary Symp., 14, 169-174, 1981.

Mukai, T., Cometary dust and interplanetary particles, in Evolution of Interstellar Dust and Related Topics, edited by A. Benti, J. M. Greenberg, and S. Aiello, pp. 397-445, Elsevier Sci. Publ. Amsterdam, 1989.

Mukai, T., H. Ishimoto, T. Kozasa, J. Blum, and J. M. Greenberg, Radiation pressure forces of fluffy porous grains, Astron. Astrophys., 262, 315-320, 1992.

Paddack, S. J. and J. W. Rhee, Rotational bursting of interplanetary dust particles, in Interplanetary Dust and Zodiacal Light, edited by H. Elsaesser and H. Fechtig, pp. 453-457, Lecture Notes in Physics 48, SpringerVerlag, Berlin-Heidelberg-New York, 1976.

Purcell, E. M. and C. R. Pennypacker, Scattering and absorption of light by nonspherical dielectric grains, Astrophys. J., 186, 705-714, 1973.

Reach, W. T., B. A. Franz, and J. L. Weiland, The three-dimensional structure of the zodiacal dust bands, Icarus, 127, 461-484, 1997.

Spiesman, J. W., G. M. Hauser, T. Kelsall, M. C. Lisse, H. S. Moseley, T. W. Reach, F. R. Sillverberg, W. S. Stemwedel, and L. J. Weiland, Near- and far-infrared observation of interplanetary dust bands from the COBE diffuse infrared background experiment, Astrophys. J., 442, 662667, 1995.

Voshchinnikov, N. V. and V. B. Il'in, Radiation pressure on cylindrical particles, Opt. Spectrosc., 55, 304-306, 1983.

Md. A. Saklayen and T. Mukai (e-mail: mukai@kobe-u.ac.jp) 